\section{Fluoride varnish application}

Sir, I am a newly qualified dentist and despite my limited experience I have noticed different opinions on when to apply fluoride varnish. Delivering better oral health guidelines ${ }^{1}$ recommend that all children should have fluoride varnish applied at least twice yearly, including even those at low caries risk, whilst children with known caries risk factors are recommended additional applications. However, there are certain medical contraindications specified by SDCEP guidelines ${ }^{2}$ and manufacturers.

I have noticed two different approaches to application of fluoride varnish, the first in which every child that attended for an examination appointment had fluoride varnish applied providing there were no co-operation issues, a specific request by the patient or guardian requesting not to apply fluoride varnish or in the case of a medical contraindication (checked by asking the patient and their accompanying guardian). However, during my dental core training year in a community dental setting I was advised that fluoride varnish could not be applied to children under 16 unless parental consent is obtained prior. Fluoride varnish application was therefore much lower as many children attended regular dental examinations with grandparents or siblings etc.

I understand that there has been a documented case of a Type 1 hypersensitivity reaction to colophony which is present within Duraphat varnish in which the patient developed allergic contact stomatitis. ${ }^{3}$ However, the patient in question had a known allergy to sticking plasters and therefore had a known risk of having an allergy to colophony so wouldn't have had fluoride varnish applied under current SDCEP recommendations. A recent randomised controlled trial conducted in South Wales involving 1,016 children aged 6-7 years old recruited over 2011-13 followed up over a 36-month period by Chestnutt et al. concluded that six-monthly applications of fluoride varnish on all surfaces of caries-free first permanent molars resulted in a caries preventative effect that is not significantly different to placement and maintenance of fissure sealants on the occlusal surfaces of caries-free first permanent molars in children. ${ }^{4}$ Fluoride varnish also exhibits advantages such as being quick and easy to apply and a less complex intervention than fissure sealants whilst not requiring any maintenance. It is due to the above reasons I believe perhaps we should be considering the use of fluoride varnishes that are colophony free or booking patients in solely for application of fluoride varnish once clarification of medical history from a parent and parental consent has been obtained.

\section{R. Khehra, South Wales}

1. Public Health England. Delivering better oral health: an evidence-based toolkit for prevention. 3rd ed. London: Public Health England, 2014.

2. Scottish Dental Clinical Effectiveness Programme. Prevention and management of caries in children. Scottish Dental Clinical Effectiveness Programme, 2010.

3. Sharma P R. Allergic contact stomatitis from colophony. Dent Update 2006; 33: 440-442.

4. Chestnutt I G, Playle R, Hutchings $S$ et al. Fissure seal or fluoride varnish? A randomized trial of relative effectiveness. J Dent Res 2017; 96: 754-761.

DOI: $10.1038 /$ sj.bdj.2018.87

\section{Radiology}

\section{New regulations}

Sir, the Ionising Radiations Regulations (IRR) were revised in 2017. The new IRR17 came into force on 1 January 2018 and will replace IRR99. One of the main changes is that dentists who have $\mathrm{X}$-ray equipment have to register with the Health and Safety Executive. Registration is carried out online and the dentist will have to answer a series of questions confirming they are complying with the Regulations. Registration opened on 3 January 2018 and dentists must have been registered by 5 February 2018. There is a fee of $£ 25$ for registration. An employer needs to register only once even if they own more than one practice containing multiple pieces of diagnostic X-ray equipment. Once registered they do not have to register again unless there is a material change in the practice. Further information is available on the HSE website.

S. Ng, President, British Society of Dental and Maxillofacial Radiology DOI: 10.1038/sj.bdj.2018.88

\section{Patient safety}

\section{Preventing aspiration}

Sir, I read with great interest the letter by V. A. Argent regarding swallowed objects (BDJ 2017; 223: 746). I would like to share a few safety measures which can prevent the accidental ingestion of brackets or a small piece of arch wire during orthodontic therapy. First, the orthodontist can use a 'throat shield' which is a small piece of gauze $(5 \times 5 \mathrm{~cm})$ unfolded and spread over the tongue and posterior area of the mouth which can prevent accidental swallowing of small objects. Secondly, a dental device known as 'Isolite' (Isolite Systems, USA) can be used which simultaneously delivers continuous throat protection, illumination, retraction, and isolation. It has a unique soft, flexible mouthpiece, which prevents the aspiration of foreign objects, retracts and protects the soft tissues, delivers illumination without casting shadows, continuously aspirates fluids, and isolates maxillary and mandibular quadrants simultaneously. The mouthpiece is disposable and hence there is no risk of cross infection, it is easy to place and it is comfortable to the patient. By using these techniques the orthodontist can prevent accidental aspiration of materials by the patient.

N. Vasudev Ballal, Jothi Varghese, Manipal, India DOI: 10.1038/sj.bdj.2018.89 